\title{
Nationale und föderale Systeme im Vereinten Europa: Brauchen wir eine Harmonisierung der Aus- und Weiterbildung in der Dermatologie?
}

\author{
National and Federal Systems in a United Europe: Do We Need Harmonisation \\ for Undergraduate and Postgraduate Training in Dermatology?
}

Autor

Institut

\section{H. P. M. Gollnick}

Universitätsklinik für Dermatologie und Venerologie, Otto-von-Guericke-Universität Magdeburg

\section{Bibliografie}

Dol $10.1055 / \mathrm{s}-0028-1119595$

Akt Dermatol 2009; 35:

115-119 @ Georg Thieme

Verlag KG Stuttgart · New York ISSN 0340-2541

Korrespondenzadresse

Prof. Dr. Harald P. M. Gollnick Universitätsklinik für

Dermatologie und Venerologie Otto-von-Guericke-Universität Leipziger Str. 44 39120 Magdeburg harald.gollnick@med.ovgu.de

\section{Zusammenfassung \\ $\nabla$}

Innerhalb der europäischen Union existiert kein einheitliches Curriculum der Medizinerausbildung. Bedauerlicherweise ist auch die fachärztliche Weiterbildung in Europa zersplittert, unterschiedlich akzentuiert, aber im Vergleich zum Medizinstudium auf dem Weg zu einer rascheren Harmonisierung. Freie Berufswahl, freie Bewe-

Die Europäische Union als Zusammenschluss von 27 hoheitlichen Ländern ist das Ergebnis eines bereits über 50 Jahre dauernden Prozesses. Es war und ist der Wunsch weitdenkender Politiker und besonders die Vernunft der Vor- und Nachkriegsgenerationen, nach einem Europa zu streben, in dem wir friedlich miteinander leben können und wollen, damit die Schrecken von Weltkriegen und politische extremistische Strömungen nie wieder diesen Kontinent erschüttern. Der erste Schritt hierzu war, die wirtschaftlichen Grenzen niederzureißen, was größtenteils und im letzten Jahrzehnt - bedingt durch die zunehmende Globalisierung - auch durch äußeren Druck weiter vorangetrieben wird. Die größten Schwierigkeiten bestehen in der Harmonisierung der sozialen Systeme und der Steuersysteme. Erstaunlicherweise gelingt erst in den letzten Jahren, die Harmonisierung der Curricula in den verschiedenen beruflichen Ausbildungsgängen voranzutreiben. Wenn wir auch auf internationalen medizinischen Kongressen dieselbe Sprache sprechen und einander gut verstehen, Ergebnisse aus Klinik und Forschung miteinander teilen, miteinander forschen, Stipendiaten von dem einen in das andere Land gehen, so ist doch erstaunlicherweise eine Harmonisierung der medizinischen Curricula und der Weiterbildungsgänge teilweise immer noch ein Torso. gung und freie Niederlassung innerhalb der EU setzen eine Abstimmung in Hinsicht auf die curriculare Weiter- und Fortbildung voraus. Aufgrund des Subsidiaritätsprinzips in der EU liegen die Bestimmungen für Abschlüsse noch in den Händen der nationalen Regierungen. Die gegenseitige Anerkennung von Abschlüssen ist noch nicht durchgehend geregelt.

\section{Medizinstudium \\ $\nabla$}

Die curriculare Ausbildung der Studentinnen und Studenten in der Humanmedizin, aber auch in der Dental- und Veterinärmedizin ist zwischen den Ländern Europas in keiner Weise harmonisiert. In Deutschland lehren wir gemäß der Approbationsordnung der Mediziner, jedoch gibt es kleine Unterschiede in der Anzahl der Unterrichtsstunden und Betonung einzelner Fächer noch in Abhängigkeit von den Bundesländern und medizinische Fakultäten haben ihren Einfluss auf die Ausgestaltung der Vorgaben der Approbationsordnung [1]. So bestehen durchaus Verschiebungen in den Proportionen zwischen Hauptvorlesung, Praktika, Seminaren und Ausbildung am Krankenbett. In Italien haben die medizinischen Fakultäten einen ganz individuellen Status in Hinsicht auf die Umsetzung des medizinischen Curriculums und es bestehen noch größere Unterschiede von Fakultät zu Fakultät, als dies in Deutschland der Fall ist. Es gibt kleinere Länder in der EU (z.B. Litauen), die parallel zu der Medizinerausbildung in der Landessprache ein Curriculum in Englisch anbieten und wo überwiegend dieselben Dozenten Studentinnen und Studenten aus allen Ländern der Welt im naturwissenschaftlich-medizinischen vorklinischen Studium ausbilden. Die Semmelweiss-Universität Budapest ist dabei, einen klinischen Studiengang in Hamburg unabhängig von der dorti- 
gen Universität einzurichten. Solche Curricula setzen voraus, dass sie in anderen Ländern Europas anerkannt werden, d.h., dass die erworbenen Zertifikate durch gegenseitige Anerkennung zwischen Fakultäten verschiedener Länder oder zwischen Ländern in Form von einem Punktesystem (Credits) akzeptiert werden.

Wenn wir das Beispiel der Dermatologie nehmen, so orientiert sich ein Lehrstuhlinhaber für Dermatologie und Venerologie überwiegend am Gegenstandskatalog, dennoch bleibt es ihm freigestellt, innerhalb der Unterrichtsstunden Themen in die eine oder andere Richtung der Gebiete des Faches zu gewichten [2]. Während in Deutschland die Dermatologie und Venerologie ein Prüfungsfach ist, welches im Studium jede/r Mediziner/-in durchläuft, haben $60 \%$ der Medizinstudentinnen und -studenten in Frankreich kaum noch Berührung mit unserem Fachgebiet. Das heißt, dass sie am Ende ihrer Ausbildung kaum oder gar nicht mit Patienten in der Dermatologie in Kontakt gekommen sind, weil Schwerpunkte anders gesetzt wurden. So kann man es durchaus erleben, dass Studenten, die während des Studiums ins Ausland gehen und z. B. im Rahmen des Erasmus-Programms Teile ihres Studiums in Frankreich ablegen und ihre dort gemachten Scheine anschließend an der Heimatfakultät vorlegen, bei der Prüfung der Inhalte, die einem Schein zugrunde liegen, weniger umfangreich Dermatologie/Venerologie vermittelt bekommen haben, als dies bei uns der Fall ist. In der Aufstellung der Lerninhalte für unser Fachgebiet spiegelt sich natürlich der Umfang der Weiterbildungsordnung wider. In Ländern, in denen die Allergologie nicht essentieller Bestandteil der Weiterbildungsordnung ist oder auch große Teile der Dermatoonkologie nicht vertreten werden, sollte man davon ausgehen, dass sie sich dann in einem anderen Teilgebiet der Medizin wiederfinden. In der Hälfte der EU-Staaten existiert die Spezialisierung für Allergologie und Klinische Immunologie, daher ist eben dieser Anteil innerhalb unseres Fachgebietes kaum oder gar nicht vertreten. Dies gilt z. B. auch für die Geschlechtskrankheiten in England und Irland.

Das Europäische Dermatologische Forum (EDF) hat es sich in jüngster Zeit zur Aufgabe gemacht, mittels einer Erhebung über die Unterrichtsstunden im Fachgebiet und den Umfang dessen, was gelehrt wird, ein gemeinsames europäisches Curriculum zu erarbeiten, damit das Fachgebiet aus dem Blickwinkel der Harmonisierung des Facharzt-Curriculums eine Basis am Ende des Studiums darstellt, auf der dann die Facharztausbildung aufbaut. Wenn wir die Bedeutung unseres Fachgebietes stabil halten wollen, müssen wir bereits im Medizinstudium unsere Ansprüche auf europäischer Ebene reklamieren [3].

In Hinsicht auf die Unterschiedlichkeit des Medizinstudiums in den europäischen Staaten zeigt sich, dass die Lehrstunden zwischen 40 bis 130 pro EU-Land für die Dermatologie schwanken. Ein nationales Lehrprogramm der Humanmedizin besteht in 11 von 23 befragten EU-Staaten (Umfrage des European Dermatology Forums, L. Dubertret, mündliche Mitteilung). 13 haben eine mündliche Prüfung, 10 schreiben Klausuren im Multiple-ChoiceSystem bzw. 11 von 23 eine schriftliche Prüfung, 10 von 23 fördern gleichzeitig zwei der drei dieser Möglichkeiten, 21 von 23 bieten ein Bedsite-Teaching an, aber schon 20 von 23 auch problemorientiertes Lernen.

\section{Was ist der Bologna-Prozess in der Humanmedizin? $\nabla$}

1999 haben 27 europäische Bildungsminister weitreichende Reformen des europäischen Bildungswesens vorgegeben. Bis zum Jahre 2010 soll die Schaffung eines zweistufigen Systems von Studienabschlüssen (Bachelor und Master) eingeführt werden. Das Europäische Credit Point-Transfer-System (ECTS) soll den Austausch von Studenten zwischen den Ländern bzw. Universitäten erleichtern. In Hinsicht auf die Medizin gab und gibt es heftige Diskussionen über die Vor- und Nachteile eines solchen zweistufigen Prinzips. Der Deutsche Medizinische Fakultätentag unterstützt das Bachelor-/Mastersystem für die Medizin nicht. Auch der 111. Deutsche Ärztetag hat 2008 die Einführung dieses Systems für die Medizin abgelehnt und den einheitlichen hochwertigen Medizinstudium-Abschluss „Staatsexamen“ weiterhin als den einzigen Abschluss des Studiums gefordert. Der Verband der leitenden Krankenhausärzte Deutschlands (VLK) lehnt ebenfalls die in einem Modellvorhaben vorgesehene Begrenzung des Zutritts zum Master-Studiengang der Medizin durch eine entsprechende Note eines Bachelor-Abschlusses ab. Hingegen sieht auch er die Förderung der Mobilität von Studenten und Ärzten durch ein europaweites Curriculum mit Anerkennung entsprechender Abschlüsse als wünschenswert an [8].

\section{Weiterbildung \\ $\nabla$}

Die Weiterbildungsordnung in der Humanmedizin in Deutschland spiegelt deutlich den föderalen Aufbau unseres Staates wider. Die Bundesärztekammer erstellt z. B. eine Musterweiterbildungsordnung [4]. Am Prozess, der letztmalig eine komplette Novellierung zwischen den Jahren 2001 bis 2004 durchlief, lässt sich die Sinnhaftigkeit eines föderalen Aufbaus mit Landesärztekammern hinterfragen. An der Musterweiterbildungsordnung arbeiten alle wissenschaftlichen Fachgesellschaften und Berufsverbände und ihre Weiterbildungskommissionen mit. In monatelangen Anhörungen werden die Grenzen der Fächer definiert und Abstimmungen zwischen den Fächern erreicht. Der Inhalt wird dann in der Musterweiterbildungsordnung verabschiedet. Diese geht dann den Landesärztekammern zu, wo wiederum in der Anhörung der Weiterbildungsausschüsse die Musterweiterbildungsordnung erneut diskutiert wird ( $\bullet$ Abb. 1, 2). Man muss hinterfragen, inwieweit die Kompetenz auf der Ebene der Landesärztekammern den höchsten Sachverstand, der bei der Erstellung der Musterweiterbildungsordnung bei der Bundesärztekammer vorhanden ist, überhaupt erreichen kann. So wurden zum Teil während der Anhörungen in den Landesärztekammern Denkprozesse wieder angestoßen, die in der Anhörung bei der BÄK schon lange abgeschlossen waren, wodurch letztlich unnötige zeitliche Verzögerungen eintraten. Man sollte annehmen, dass die aus der Deutschen Dermatologischen Gesellschaft und dem Berufsverband der Deutschen Dermatologen gewählten Vertreter das entsprechende Mandat haben, um die Belange des Faches bei der BÄK und in den Anhörungen zu vertreten und nicht auf der lokalen Ebene der Länder die in diesem langen Anhörungsprozess errungenen Formulierungen und Inhalte wieder zu hinterfragen, die wieder zum Teil von Partikularinteressen getragen wurden.

Auf der europäischen Ebene ist man seit der Gründung der Union of Mecical Specialists (Vereinigung der Fachärzte Europas/UEMS) 1954 bestrebt, zum einen die Ansprüche der Fachärz- 


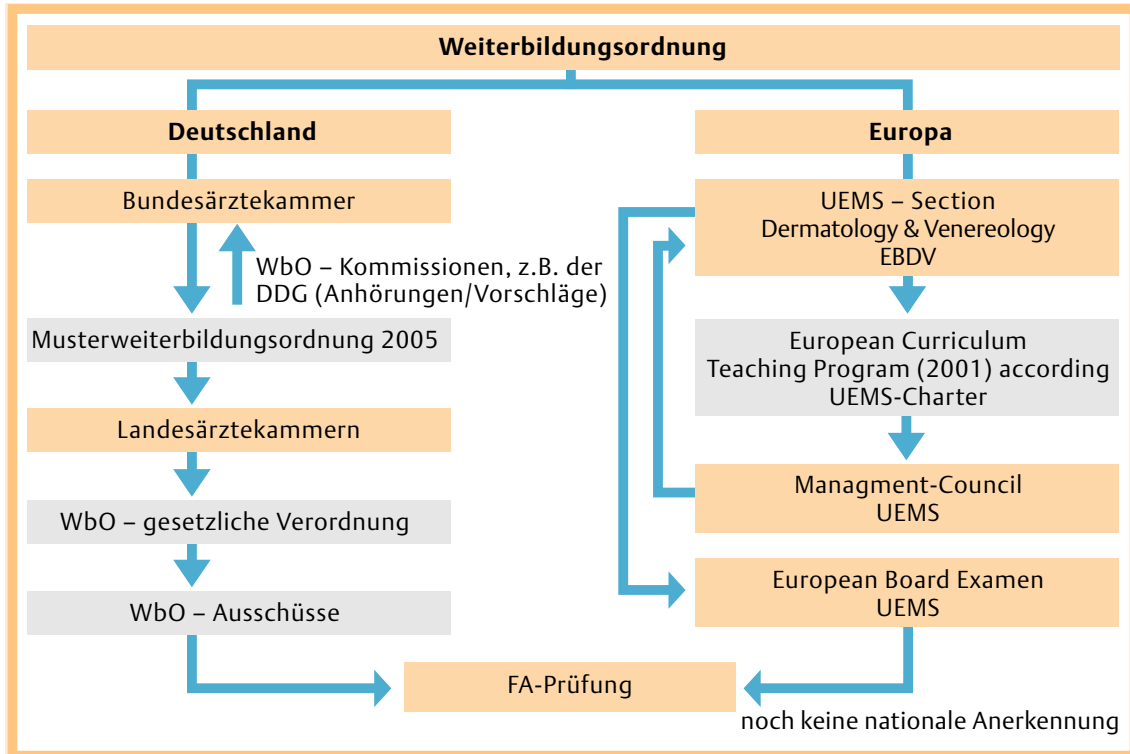

Abb. 1 Vereinfachte Übersicht zum Ablauf der Entwicklung von Weiterbildungsordnungen in Deutschland und Europa.

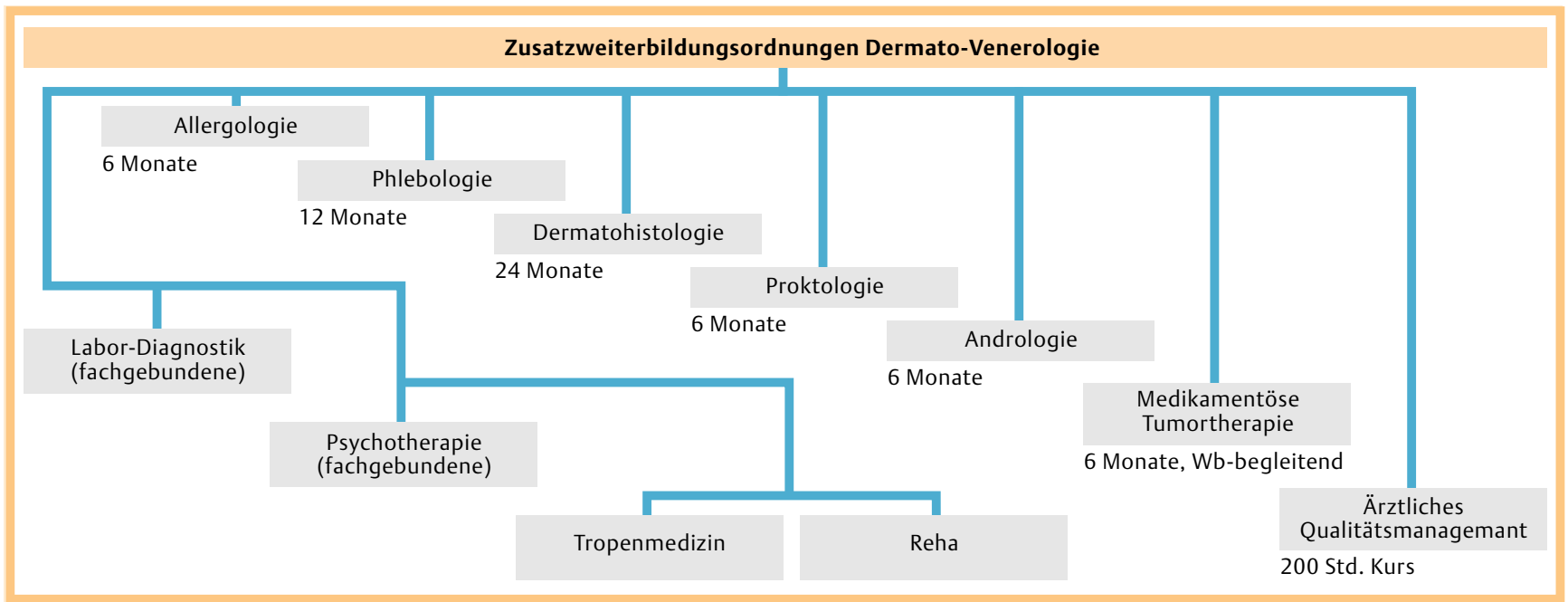

Abb. 2 Wichtige Zusatzweiterbildungen für das Fachgebiet Dermato-Venerologie.

te in der Humanmedizin in ihrem beruflichen Umfeld zu vertreten und zum anderen - getragen von dem UEMS-Charter Curriculum Teaching Programm - eine Harmonisierung zu erreichen [5]. In der UEMS ist für jede medizinische Disziplin eine Sektion eingerichtet, so auch die Section of Dermato-Venereology/European Board of Dermato-Venereology (EBDV). Letztmalig wurde das Curriculum der Dermato-Venerologie 2001 von der Sektion verabschiedet, welches in den Grundzügen der Deutschen Weiterbildungsordnung entspricht. Damit wird der Anspruch der deutschsprachigen Dermatologie Deutschlands, Österreichs und der Schweiz in besonderer Weise festgeschrieben. Es bestehen innerhalb Europas große Unterschiede zwischen den Ländern, was die Abbildung von Spezialgebieten unseres Faches wie der Allergologie, der Dermatoonkologie, der Phlebologie, der Andrologie, der Proktologie und der operativen Dermatologie betrifft. Ebenso variieren die Weiterbildungszeiten, die zwischen 3 und 5 Jahren betragen, zum Teil auch abhängig davon, ob der Ausbildung zum Facharzt für Dermatologie ein sogenannter Common Trunc mit einem Jahr Innere Medizin und einem Jahr Chirurgie oder einem Jahr Chirurgie und Innere Medizin vorgeschaltet ist. Die akademischen Lehrer unseres Fachgebietes haben erkannt, dass zum Erhalt unseres Faches eine möglichst komplette Abbildung der Inhalte erreicht werden soll. Hierzu wurden zwei Wege eingeschlagen: Der eine zielt darauf hin, dass in Ländern, in denen das Fach nicht so komplett abgebildet ist wie in der deutschsprachigen Dermatologie, die Ansprüche auf die eben genannten Teilgebiete mehr und mehr verfolgt werden sollen, der andere ergänzende Weg ist das europäische Facharztexamen Dermato-Venerologie. Nach über zweijähriger Vorbereitung durch eine Subkommission der Sektion DermatoVenerologie der UEMS konnte ein 400 Prüfungsfragen umfassendes Examen erstellt werden, welches 2007 erstmalig in Frankfurt/Main etabliert wurde. Das Examen dauerte von Freitagmittag bis Sonntagmittag und umfasst alle Kern- und Teilgebiete unseres Faches. Das zweite Examen fand erfolgreich im August 2008 statt und wird erneut im Sommer 2009 durchgeführt werden. Das hierbei zu erreichende Diplom hat keinen rechtlichen Status, der einen Anspruch ergibt, dass das Diplom das Facharztexamen in einem anderen europäischen Land wegen dessen Aufsichtshoheit ersetzt ( $\bullet$ Abb. 1). Innerhalb Europas gibt es Staaten, die gar kein Examen abnehmen und nur die jährlichen Logbuchkontrollen und individuellen jährlichen Zwi- 
schenprüfungen am Ende der Weiterbildungszeit zur Zulassung/ Spezialgebietanerkennung führen, oder Länder, in denen wie z.B. in Deutschland nur eine formale 30- bis 45-minütige Prüfung bei der Landesärztekammer erfolgt, oder Länder wie Schweiz und Österreich ein ganz- oder mehrtägiges Examen. Für Kolleginnen und Kollegen aus Ländern, deren Curriculum sich nicht voll im europäischen Curriculum widerspiegelt, ist jedoch das europäische Examen ein Anreiz, für sich selbst zu prüfen, was sie können und was sie durch zusätzliches Lernen erreicht haben. Hierzu dient auch das jährlich über jeweils $4 \frac{1}{2}$ Tage stattfindende Euroderm Excellence Meeting der EDF in Rom, bei dem 150 Weiterbildungsassistentinnen/-assistenten im letzten Ausbildungsjahr noch einmal das gesamte Fachgebiet von erfahrenen akademischen Lehrern in Vorlesungen und Diskussionen dargeboten bekommen. Dieses stellt dann eine sehr gute Vorbereitung für das Europäische Board Examen dar. Die Allergologie als wichtiges Teilgebiet unseres Faches ist in der Hälfte der Länder der EU mit einer Superspezialisierung nach der Facharztweiterbildung verbunden. In anderen EU-Ländern gibt es das Fachgebiet der Allergologie und Klinischen Immunologie. Die Sektion Allergologie der UEMS erhebt jedoch den Anspruch, dass nur Internisten und Pädiater das vollständige Curriculum durchlaufen können, sodass die Dermatologen und andere Fächer von dieser Ausbildung weitgehend abgeschnitten sind. Seit 2003 hat es sich die UEMS-Sektion Dermatologie zusammen mit anderen Sektionen wie Kinder-Pneumologie, ErwachsenenPneumologie, HNO, Rheumatologie, Neurologie, Gastroenterologie und experimentelle Immunologie zur Aufgabe gemacht, einen Widerpart zu bilden. 2005 wurde vom Management Conncil der UEMS die Einrichtung eines Interdisciplinary Joint Committee for Immune Mediated Disease eingerichtet. Hiermit soll es zu einer curriculären Übereinstimmung der Weiterbildungsinhalte auf diesem Gebiet kommen, damit auch in den Ländern, in denen den Dermatologen der Weg nicht primär offen steht, eine Ausbildung gewährleistet werden kann. Deutschland war das erste Land, in dem die Zusatzweiterbildung „Dermatohistologie“ zwischen den Fachgebieten Dermatologie und Pathologie konsensuell verabschiedet wurde. Inzwischen besteht die Möglichkeit ebenfalls in Österreich und der Schweiz ( $\bullet$ Abb. 2, 3). Seit 2003 wurde unter der Leitung des International Committee of Dermatopathology (ICDP) in Frankfurt am Main das Examen für Dermatopathologie eingerichtet. Es war das Bestre-

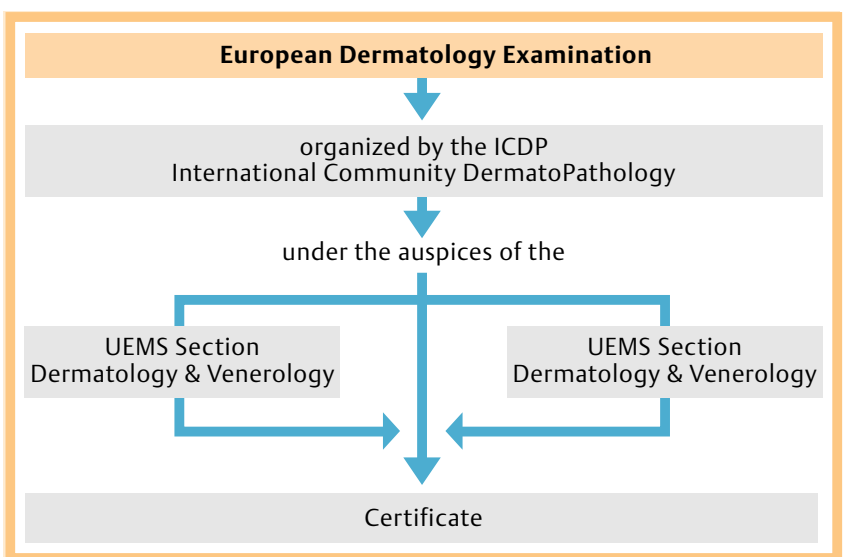

Abb. 3 Europäisches Boardexamen Dermatologie \& Venerologie.

ben der UEMS-Sektion Dermatologie die Sektion Pathologie davon zu überzeugen, dass die Dermatopathologie eine Besonderheit darstellt und es einer speziellen Ausbildung nicht nur für Dermatologen, sondern auch für Pathologen bedarf. Diese langwierige Diskussion hat zu dem erfolgreichen Abschluss geführt, dass dieses Examen nun unter der Schirmherrschaft der ICDP zusammen mit den beiden UEMS-Sektionen jährlich abgenommen wird und das Diplom von diesen Dreien gemeinsam vergeben wird. Darüber hinaus haben beiden Sektionen zusammen mit der ICDP im Dezember 2008 eine Roadmap erfolgreich verabschiedet, die die curriculäre Weiterbildung Dermatopathologie in Europa harmonisiert.

Um die Qualität der Weiterbildung an den Kliniken zu prüfen, besteht die Charter of Visitation der UEMS [6]. Andere Fachgebiete haben daher schon seit Jahren regelmäßig Visitationen an Ausbildungsstätten durchgeführt, um mit den Weiterbildungsassistentinnen/-assistenten deren Ausbildung vor Ort zu diskutieren, die minimalen Ausbildungsbedingungen zu definieren, die Ausrüstung der Kliniken zu prüfen, die Logbücher zu harmonisieren und somit zwischen den EU-Ländern einen Gleichstand zu erreichen [7].

Weiterhin ist festzustellen, dass in der Dermato-Venerologie, aber auch in der Frauenheilkunde und Geburtshilfe, der HalsNasen- und Ohrenheilkunde und der Urologie seit 2003 die

Tab. 1 Anerkennung von Facharztbezeichnungen (Quelle: Bundesärztekammer).

\begin{tabular}{|c|c|c|c|c|c|c|c|c|c|c|}
\hline \multirow{2}{*}{$\begin{array}{l}\text { Facharzt } \\
\text { bezeich- } \\
\text { nung }\end{array}$} & \multicolumn{2}{|l|}{2003} & \multicolumn{2}{|l|}{2004} & \multicolumn{2}{|l|}{2005} & \multicolumn{2}{|l|}{2006} & \multicolumn{2}{|l|}{2007} \\
\hline & $\begin{array}{l}\text { Anzahl } \\
\text { insg. }\end{array}$ & $\begin{array}{l}\text { darunter: } \\
\text { Ärztinnen }\end{array}$ & $\begin{array}{l}\text { Anzahl } \\
\text { insg. }\end{array}$ & $\begin{array}{l}\text { darunter: } \\
\text { Ärztinnen }\end{array}$ & $\begin{array}{l}\text { Anzahl } \\
\text { insg. }\end{array}$ & $\begin{array}{l}\text { darunter: } \\
\text { Ärztinnen }\end{array}$ & $\begin{array}{l}\text { Anzahl } \\
\text { insg. }\end{array}$ & $\begin{array}{l}\text { darunter: } \\
\text { Ärztinnen }\end{array}$ & $\begin{array}{l}\text { Anzahl } \\
\text { insg. }\end{array}$ & $\begin{array}{l}\text { darunter: } \\
\text { Ärztinnen }\end{array}$ \\
\hline $\begin{array}{l}\text { Frauenheil- } \\
\text { kunde u. } \\
\text { Geburtshilfe }\end{array}$ & 652 & 464 & 626 & 459 & 627 & 471 & 617 & 469 & 574 & 456 \\
\hline $\begin{array}{l}\text { Hals-Nasen- } \\
\text { Ohrenheil- } \\
\text { kunde }\end{array}$ & 204 & 72 & 170 & 58 & 193 & 70 & 200 & 81 & 210 & 98 \\
\hline $\begin{array}{l}\text { Haut- und } \\
\text { Geschlechts- } \\
\text { krankheiten }\end{array}$ & 242 & 149 & 218 & 139 & 234 & 145 & 224 & 143 & 200 & 140 \\
\hline Urologie & 212 & 31 & 244 & 28 & 212 & 32 & 227 & 46 & 200 & 37 \\
\hline
\end{tabular}


Zahl der Facharztanerkennungen stagniert oder sogar abnimmt. In Anbetracht der Tatsache, dass bereits jetzt und noch mehr in den kommenden 5 Jahren die Zahl der Fachärzte in der Niederlassung aufgrund eines Generationenwechsels um etwa 10\%abnehmen wird, ist dies auf der einen Seite eine politische Herausforderung, die Bedingungen des Arztberufs in der Freien Praxis zu verbessern, die Stellenpläne für Weiterbildungsassistenten/innen an den Kliniken anzuheben und auf der anderen Seite durch die Harmonisierung und gegenseitige Anerkennung von Facharztabschlüssen aus anderen EU-Ländern - wie oben bereits gesagt - dringend voranzutreiben, um die freie Niederlassung innerhalb Europas zu gewährleisten und auch ausländische Kolleginnen und Kollegen nach Deutschland attrahieren zu können ( $\bullet$ Tab. 1).

Es ist davon auszugehen, dass der Kommissar für Ausbildungsfragen bei der EU in der nächsten Zeit harmonisierte curriculäre Ausbildungen in der Medizin hinterfragen wird. Harmonisierungen für Gebiete wie die Ausbildung zum Chemiker, Agraringenieur und andere sind dabei schon weiter fortgeschritten. Die Dermato-Venerologie tut gut daran, ihre Harmonisierung in Europa tatkräftig voranzutreiben. Dabei wird in der Zukunft die Bundesärztekammer Ansprechpartner auf der nationalen Ebene sein und nicht mehr die Landesärztekammern. Spätestens dann wird man sich fragen, inwieweit in einem gemeinsamen Europa noch die Aufsichtsfunktion der LÄKs auf föderaler Länderebene weiter bestehen kann.

\section{Abstract}

\section{National and Federal Systems in a United Europe: \\ Do We Need Harmonisation for Undergraduate and Postgraduate Training in Dermatology? \\ $\nabla$}

Undergraduate training in medicine within the European Union is almost lacking harmonisation. Moreover, specialists' training in Dermato-Venerology is also not performed under an umbrella of harmonisation in the EU, however, a European curriculum teaching program is published under the auspices of the UEMS Section Dermato-Venereology, and, a harmonized program for Dermato-Pathology as well. The acknowledgement of diplomas in medicine is still biased due to the subsidiarity principle and the national authority in the health system of EU-countries.

\section{Literatur}

1 Bundesärztekammer. Ärztliche Ausbildung, Weiterbildung und Fortbildung in Tätigkeitsbericht 2005 der Bundesärztekammer. Köln: Deutscher Ärzteverlag, 2005: $341-365$

2 2. ÄP Schwerpunkt Urologie \& Dermatologie. Prüfungsfragen mit Kommentar. Einzelfragen und Fallstudien. Schwarze Reihe. Stuttgart: Thieme, 2008

3 Fritsch P, Burgdorf W (eds). EDF Whitebook. Skin Diseases in Europe. European Dermatology Forum. Berlin: ABW-Wissenschaftsverlag, 2005

4 Muster-Weiterbildungsordnung 2003 der Bundesärztekammer. Arbeitsgemeinschaft der Deutschen Ärztekammern, Fassung vom 28.3.2008

Im Internet: www.bundesaerztekammer.de

5 Gollnick H. Status of Dermatology in Europe. Union Européenne des Médecins Specialistes/European Union of Medical Specialists (UEMS)/ Section and Board of Dermatology and Venerology. In: Fritsch P, Burgdorf W (eds). EDF Whitebook, 2005: 215-218

6 Fras Z, Destrebecq F. The UEMS 50 Years Anniversary Book. Brüssel: Grafimmo, 2008

7 Rosdahl I, Finaly A, Gollnick H, Lomutao M. Soyland E. Guidelines for character on visitation of training centres in dermatology and venereology: report for the European Board of Dermatology and Venereology, European Union of medicals specialists. J Eur Acad Dermatol Venereol 2001; 15: 272 - 279

8 VLK (2009). Arzt und Krankenhaus, 82 (1): 4-6 